\title{
Consistent Algorithm for Tensegrity from Form-finding to Folding and Deployment Simulation with Common Scheme
}

\author{
Cho Kyi Soe*, S. Yamashita ${ }^{\dagger}$, E. Krasniqi ${ }^{\dagger}$, K. Ijima ${ }^{\dagger}$ and H. Obiya ${ }^{\dagger}$ \\ * Graduate School of Science and Engineering \\ Saga Univeristy, Japan \\ 1 Honjo Saga, 840-8502, Japan \\ E-mail: chokyisoe@gmail.com \\ $\dagger$ Department of Civil Engineering \\ Saga University, Japan \\ 1 Honjo Saga, 840-8502, Japan \\ Email: obiyah@cc.saga-u.ac.jp
}

\begin{abstract}
In recent decades, tensegrity has gained its popularity due to its mysterious geometric morphology and significant characteristics. Tensegrity is a structure composed of disconnected struts or bars enclosed in a network of tensile cables. These structures are light in weight and they maintain their stability by the self-equilibrium [1]. The unique behaviour of tensegrity structures have been investigated in different ways for years according to the purpose of analyses.

In this paper, we developed an algorithm which can consistently conduct from form-finding process using virtual elements to static/dynamic simulation process of folding and deployment with hyperelastic elements. This algorithm provides both processes by common scheme of the tangent stiffness method which is quite effective in the geometrical nonlinear analysis due to its strict rigid body displacement of elements [2]. The algorithm has more rationally than in case using the force density method in form-finding process.
\end{abstract}

A simple tensegrity structure is proposed as a numerical model and its equilibrium configuration is determined by the form-finding analysis. The obtained equilibrium shape is made as a simulation model and each member of the model is then substituted by the real element respectively. The stiffer element (strut) is designated as a linear steel rod and the softer element (cable) is assigned by the application of hyper-elastic material.

In this study, Ogden model is used as a basic function with the combination of square-root function to characterize the hyper-elastic behaviour to a full extent. The folding simulation is conducted under the given compulsory displacement and the change in length and axial force is examined in each stage. The dynamic analysis is carried out to describe the movement of elements during the folding process. Our proposed method explains the dynamic behaviour along with the folding simulation of the proposed tensegrity structure.

\section{REFERENCES}

[1] H. Furuya, "Concept of deployable tensegrity structures in space application", Int. J. Space Structures, 7, 143-151, (1992).

[2] A. Matsuo, H. Obiya, K. Ijima and Z. M. Nizam, "Form-finding of extensive tensegrity using truss elements and axial force lines", Int. Conf. on Textile Composites and Inflatable Structures, Structural Membranes, Barcelona, Spain (2011). 